\title{
Representations of Unitary Groups and Free Convolution
}

By

\author{
Philippe BIANE*
}

\begin{abstract}
To each finite dimensional representation of a unitary group $U(n)$ is associated a probability measure on the set of integers, depending on the highest weights which occur in this representation. We show that asymptotically for large $n$ and large irreducible representations of $U(n)$ the measure associated to the tensor product of two representations, or to the restriction of a representation to a subgroup $U(m)$ with $m$ comparable to $n$, can be expressed in terms of the measures associated to the first representations by means of the notion of free convolution (namely additive free convolution for the tensor product problem and multiplicative free convolution for the restriction problem).
\end{abstract}

\section{Introduction}

Let $U(n)$ be the group of complex $n \times n$ unitary matrices. In the representation theory of this group two basic questions are the following.

i) Given an irreducible representation $R$ of $U(n)$ and $m \leq n$, decompose the restriction of $R$ to $U(m)$ (embedded as a subgroup of $U(n)$ ) into irreducible components.

ii) Given two irreducible representations $R_{1}$ and $R_{2}$ of $U(n)$ decompose the (Kronecker) tensor product representation $R_{1} \otimes R_{2}$ into irreducible components.

The first problem can be dealt with by using recursively Weyl's branching rule, which says that given an irreducible representation of $U(n)$ with highest weight $\left(v_{1}, \ldots, v_{n}\right)$ (where $v_{j}$ for $1 \leq j \leq n$ are integers and $v_{1} \geq v_{2} \geq \cdots \geq v_{n}$ ), then in the restriction of $R$ to $U(n-1)$ only the irreducible representations with highest weights $\left(\lambda_{1}, \ldots, \lambda_{n-1}\right)$ satisfying $v_{1} \geq \lambda_{1} \geq v_{2} \geq \cdots \geq \lambda_{n-1} \geq v_{n}$ appear, each with multiplicity 1 . From this one can determine the multiplicity $a_{l, \lambda}^{n, v}$ of the dominant weight $\lambda$ in the restriction of the representation of $U(n)$ with highest weight $v$ to $U(l)$.

Communicated by M. Kashiwara, January 25, 1994.

1991 Mathematics Subject Classifications: 22E46.

* C.N.R.S. Laboratoire de Probabilités, Tour $563^{e}$ étage, Université Paris 6, 4, place Jussieu 75252 Paris Cedex 05, France. 
For the second problem, Weyl's character formula for representations of semi-simple Lie groups can be used to give an explicit formula for the multiplicity $a_{\mu \nu}^{\lambda}$ with which the dominant weight $\lambda$ occurs in a tensor product of two irreducible representation with highest weights $\mu$ and $v$ (see [6]). In the case of unitary groups the Littlewood-Richardson rule provides another approach to this problem (see [4]).

In this paper we shall be interested in asymptotic results on these problems when both the dimension of the group and the representations become large (where for an irreducible representation to be large we mean that the components of its highest weight become large). In this case the explicit formulas for the multiplicities in both problems become quickly intractable and one has to find other means of obtaining information on them. One way to do this is to try to estimate the asymptotic behaviour of some linear combinations of the coefficients. This is what we do in this paper using the notion of free convolution of measures, introduced by $\mathrm{D}$. Voiculescu in the context of operator algebras (see [1]).

We will associate to each finite dimensional representation of a unitary group a measure on the set of integers $\mathbb{Z}$ in the following way: if $R$ is an irreducible representation of $U(n)$ then the measure associated to $R$ is $\mathscr{M}(R)=$ $\frac{1}{n} \sum_{k=1}^{n} \delta_{\mu_{k}}$ where $\delta_{x}$ is the Dirac measure at $x$ and $\mu_{k} \in \mathbb{Z}$ are the components of the highest weight of $R$. If $R=R_{1} \oplus R_{2}$ decomposes into a direct sum of representations then $\mathscr{M}(R)$ is the convex combination $\mathscr{M}(R)=\frac{\operatorname{dim}\left(R_{1}\right)}{\operatorname{dim}(R)} \mathscr{M}\left(R_{1}\right)$ $+\frac{\operatorname{dim}\left(R_{2}\right)}{\operatorname{dim}(R)} \mathscr{M}\left(R_{2}\right)$.

When the representation $R$ is irreducible the measure $\mathscr{M}(R)$ determines completely its isomorphism class. When $R$ is not irreducible, although this measure does not in general determine the decomposition of $R$ into irreducible components, it gives some partial information on the multiplicities of the irreducible representations which occur in $R$.

We will show that asymptotically the map $\mathscr{M}$ converts the tensor product of representations into the additive free convolution of measures, and that the operation of restriction to a subgroup can be expressed through $\mathscr{M}$ with the help of the multiplicative free convolution by a measure.

Informally the results that we prove can be expressed as follows: denote by $\mathscr{M}^{\varepsilon}(R)$ the image of the measure $\mathscr{M}(R)$ by the transformation $x \mapsto \varepsilon x$ on $\mathbb{R}$ for a small $\varepsilon \in \mathbb{R}_{+}$, and let $R_{1}$ and $R_{2}$ be two irreducible finite dimensional representations of $U(n)$. If $n$ is large, such that $\mathscr{M}^{\varepsilon}\left(R_{1}\right)$ and $\mathscr{M}^{\varepsilon}\left(R_{2}\right)$ are close to some probability measures $\alpha_{1}$ and $\alpha_{2}$ with compact support on $\mathbb{R}$, then $\mathscr{M}^{\varepsilon}\left(R_{1} \otimes R_{2}\right)$ is close to the measure $\alpha_{1} \oplus \alpha_{2}$ which is the free convolution of $\alpha_{1}$ and $\alpha_{2}$. In the same vein we will have that if $m$ is an integer close to 
$p n$ for $p \in] 0,1]$ then $\mathscr{M}^{\varepsilon}\left(R_{1 \mid U(m)}\right)$ (where $\left(R_{1 \mid U(m)}\right.$ is the restriction of $R_{1}$ to $U(m))$ is close to the measure $\frac{1}{p}\left(\alpha_{1} \otimes\left(p \delta_{1}+(1-p) \delta_{0}\right)-(1-p) \delta_{0}\right)$ where $\otimes$ denotes the multiplicative free convolution of measures, and $\delta_{x}$ the Dirac measure on $x \in \mathbb{R}$.

The proof of these results will consist in a two-step approximation argument.

In the first step one shows that, for fixed $n$, and for an irreducible representation $R$ of $U(n)$ with a large highest weight $\left(\mu_{1}, \ldots, \mu_{n}\right)$, the operators $d R\left(E_{k l}\right)$ (where $d R$ is the corresponding representation of the complexified Lie algebra $g l_{n}(\mathbb{C})$ and $E_{k l}$ is the canonical basis of $g l_{n}(\mathbb{C})$ ), considered as non-commutative random variables, can be approximated in distribution by the components of a random matrix with spectrum close to $\left\{\mu_{1}, \ldots, \mu_{n}\right\}$. This is just a simple consequence of Kirillov's formula for the character of an irreducible representation. In fact the result that we prove could be extended to arbitrary compact semi-simple Lie groups.

The second step consists in considering a matrix canonically associated to any representation of $U(n)$ by the formula $C(R)=\sum_{k l} E_{k l} \otimes d R\left(E_{k l}\right)$. Using the result of the first part of the paper, as well as results of $D$. Voiculescu and R. Speicher on asymptotic freeness of large independent matrices, we show that the matrices $C(R)$ corresponding to independent representations (i.e. acting on different components of a tensor product space) are asymptotically free random variable with distribution close to $\mathscr{M}(R)$, and are also asymptotically free with diagonal matrices. This asymptotic freenes allows us to link the decomposition problems for representations with free convolution.

In fact our results show how to construct in a natural way families of non-random matrices which are asymptotically free random variables with prescribed distributions.

This paper is organized as follows. In the first part, we recall some definitions and results and establish the notations. In the second part we use Kirillov's character formula to give an estimate which relates traces of some operators in an irreducible representation of $U(n)$ with the integrals of products of coordinate functions on the orbit of the highest weight in the coadjoint representation. In the third part, we introduce the probability measure and the $C$-matrix associated to a representation of $U(n)$ and give some of their properties, then we prove the main result of the paper, and we deduce from this the results concerning the tensor product and the restriction problem. Finally in the end we treat an explicit example.

\section{$\S 1$}

We recall here several definitions and notations.

1.1. First we deal with free families and free convolution (see [1] for more details). 
A non-commutative probability space is a couple $(\mathscr{A}, \phi)$ where $\mathscr{A}$ is a unital $*$-algebra and $\phi$ a positive linear functional on $\mathscr{A}$ such that $\phi(1)=1$. The elements of $\mathscr{A}$ are called non-commutative random variables. Let $\mathbb{C}\left\langle y_{l}, y_{l}^{*}\right\rangle_{l \in I}$ be the free algebra generated by the symbols $y_{l}, y_{l}^{*}, l \in I$, with the involution $y_{\imath} \mapsto y_{i}^{*}$.

The law (or distribution) of a family $\left(Y_{l}\right)_{l \in I}$ of non-commutative random variables is the linear map

$$
\begin{gathered}
D_{Y}: \mathbb{C}\left\langle y_{l}, y_{i}^{*}\right\rangle_{l \in I} \rightarrow \mathbb{C} \\
P \mapsto \phi\left(P\left(Y_{l}, Y_{l}^{*}\right)\right)
\end{gathered}
$$

where elements of $\mathbb{C}\left\langle y_{l}, y_{l}^{*}\right\rangle_{l \in I}$ are considered as non-commutative polynomials. If $\left(Y_{l}^{(n)}\right)_{l \in I}$ for $n \in \mathbb{N}$ and $\left(Y_{l}^{\infty}\right)_{l \in I}$ are families of non-commutative random variables, one says that the law of $\left(Y_{l}^{(n)}\right)_{l \in I}$ converges to that of $\left(Y_{l}^{\infty}\right)_{l \in I}$ as $n \rightarrow \infty$ if for every $P \in \mathbb{C}\left\langle y_{l}, y_{l}^{*}\right\rangle_{l \in I}$ one has $D_{Y^{(n)}}(P) \rightarrow D_{Y^{\infty}}(P)$.

A family $\left(\mathscr{A}_{l}\right)_{l \in I}$ of subalgebras of $\mathscr{A}$ is said to be free if for any $k \in \mathbb{N}$ and any sequence $a_{1} \in \mathscr{A}_{l_{1}}, \ldots a_{k} \in \mathscr{A}_{l_{k}}$ one has $\phi\left(a_{1} \ldots a_{k}\right)=0$ whenever $l_{j} \neq l_{j+1}$ for $j=1, \ldots, k-1$ and $\phi\left(a_{j}\right)=0$ for all $j=1, \ldots, k$. A family of sets of noncommutative random variables $\left(E_{l}\right)_{l \in I}$ is said to be free if the family of algebras $\left(\mathscr{A}_{l}\right)_{l \in I}$ is free where for each $l, \mathscr{A}_{l}$ is the algebra generated by the set $E_{l}$.

The law of a free family $\left(Y_{l}\right)_{l \in I}$ is determined by the law of each variable $Y_{l}$.

A sequence of families of sets of non-commutative random variables $\left(E_{l}^{(n)}\right)_{l \in I}$ with $E_{l}^{(n)}=\left\{Y_{\sigma}^{(n)} \mid \sigma \in J_{l}\right\}$ is said to be asymptotically free if the law of the family random variables $\left(Y_{\sigma}^{(n)}, \sigma \in J_{l}, l \in I\right)$ converges towards the law of some family of random variables $\left(Y_{\sigma}^{\infty}, \sigma \in J_{l}, l \in I\right)$ such that the sets $E_{l}^{\infty}=\left\{Y_{\sigma}^{\infty} \mid \sigma \in J_{l}\right\}$ for $l \in I$ form a free family of sets of random variables.

If $(X, Y)$ is a free family then the distribution of $X+Y$ depends only on the distributions of $X$ and of $Y$, it is called the additive free convolution $D_{X+Y}=D_{X} \oplus D_{Y}$. In the same way, the distribution of $X Y$ depends only on $D_{X}$ and $D_{Y}$ and is called the multiplicative free convolution denoted by $D_{X} \otimes$ $D_{Y}=D_{X Y}$. If $\mathscr{A}$ is a $C^{*}$-algebra, $X$ and $Y$ are positive elements then $D_{X} \otimes D_{Y}$ is also the distribution of $X^{1 / 2} Y X^{1 / 2}$ so that the multiplicative free convolution of two probability measures with compact supports on $\mathbb{R}_{+}$is again a probability measure with compact support on $\mathbb{R}_{+}$.

1.2. Let $U(n)$ be the group of $n \times n$ complex unitary matrices and $\mathfrak{u}(n)$ the corresponding Lie algebra of $n \times n$ anti-hermitian matrices. Let $\mathfrak{D}_{n}$ be the Lie subalgebra of diagonal matrices. The complexification of $\mathfrak{u}(n)$ is $g l_{n}(\mathbb{C})=$ $\mathfrak{u}(n)+i \mathfrak{u}(n)$, the Lie algebra of the complex group $G L_{n}(\mathbb{C})$ and $d_{n}=\mathfrak{D}_{n}+i \mathfrak{D}_{n}$ is the complexification of $\mathrm{D}(n)$. We call $\left(E_{k l}\right)_{1 \leq k, l \leq n}$ the canonical basis of $g l_{n}(\mathbb{C}) \sim M_{n}(\mathbb{C})$.

Let $\mathfrak{u}(n)^{*}$ be the dual space of $\mathfrak{u}(n)$ and $\mathfrak{D}(n)^{*}$ the dual space of $\mathfrak{D}(n)^{*}$. Let $P_{+}$be the set of dominant weights which are the elements $\mu$ of $D(n)^{*}$ such 
that $\mu\left(i E_{k k}\right) \in \mathbb{Z}$ and $\mu\left(i E_{11}\right) \geq \cdots \geq \mu\left(i E_{n n}\right)$. Every irreducible representation of $U(n)$ (or equivalently finite dimensional irreducible representation of $G L_{n}(\mathbb{C})$ ) has a highest weight with respect to the ordered basis $\left(i E_{k k}\right)_{1 \leq k \leq n}$ of $\mathfrak{D}_{n}$, which is a dominant weight and it characterizes the representation up to isomorphism. There is an inclusion $\mathfrak{D}_{n}^{*} \subset \mathfrak{u}_{n}^{*}$ dual to the canonical projection $\mathfrak{u}_{n} \rightarrow \mathfrak{D}_{n}$, and elements of $\mathfrak{u}_{n}^{*}$ can be extended to complex linear functionals on $g l_{n}(\mathbb{C})$.

For a dominant weight $\mu$ we put $|\mu|=\sup _{k}\left|\mu\left(i E_{k k}\right)\right|$. Let $\eta_{k}$ be the dual basis of $\left(i E_{k k}\right)_{1 \leq k \leq n}$ in $\mathfrak{D}(n)^{*}$ and $\rho$ be half the sum of positive roots $\rho=\sum_{k=1}^{n}(n-k+1) n_{k}$. For $v \in \mathfrak{u}(n)^{*}$ let $\mathcal{O}_{v}^{*}$ be the orbit of $v$ under the coadjoint action of $U(n)$.

Let $R_{\mu}$ be a unitary irreducible representation with highest weight $\mu$ on some Hilbert space $V$. In the non-commutative probability space $(\mathscr{L}(V), t r)$ (where $t r$ is the normalized trace on $\mathscr{L}(V)$ ) let us consider the random variables $\xi_{k l}^{\mu}=d R_{\mu}\left(E_{k l}\right)$ (where $d R_{\mu}$ is the corresponding complex representation of $g l_{n}(\mathbb{C})$ ).

The Kirillov character formula for $R_{\mu}$ (see [2]) can be stated as

$$
\operatorname{tr}\left(\exp \left(i \sum_{k l} \alpha_{k l} \xi_{k l}^{\mu}\right)\right)=\left(\prod_{k<l} \frac{\alpha_{l l}-\alpha_{k k}}{\operatorname{sh}\left(\alpha_{l l}-\alpha_{k k}\right)}\right) \int_{\mathcal{O}_{\mu+\rho}^{*}} \exp \left(i \sum \alpha_{k l} \omega\left(E_{k l}\right)\right) d \omega
$$

for complex numbers $\alpha_{k l}$ such that $\alpha_{k l}=\bar{\alpha}_{l k}$, and where $d \omega$ denotes the normalized invariant measure on $\mathcal{O}_{\mu+\rho}^{*}$.

1.3. A random matrix is a random variable of some non-commutative probability space of the form $\left(M_{n}(\mathbb{C}) \otimes L^{\infty}(\Omega, \mathscr{F}, P), \operatorname{tr} \otimes P\right)$ where $(\Omega, \mathscr{F}, P)$ is a probability space and $t r$ is the normalized trace on $M_{n}(\mathbb{C})$.

Let $\mathcal{O}_{\lambda}$ denote the orbit of the matrix $\sum_{k=1}^{n} \lambda_{k} E_{k k}$ (where $\lambda_{k} \in \mathbb{R}$ for $1 \leq$ $k \leq n)$ under the adjoint action of $U(n)$ on $i \mathfrak{u}(n)$, with its invariant probability measure. Let $Y_{k l}^{\lambda}$ denote the $k l$ coordinate function on $\mathcal{O}_{\lambda}$. A uniform random matrix with spectrum $\left\{\lambda_{1}, \ldots, \lambda_{n}\right\}$ is a random matrix $\sum_{k l} E_{k l} H_{k l}$ where the joint law of the random variables $H_{k l}, 1 \leq k, l \leq n$ is the same as the joint law of the random variables $Y_{k l}^{\lambda}, 1 \leq k, l \leq n$.

Let $v \in \mathfrak{u}^{*}(n)$, then $v$ is in the orbit of some element of $\mathfrak{D}^{*}(n)$ of the form $\sum_{k} v_{k} \eta_{k}$. Define the measurable maps $X_{k l}^{v}:\left(\mathcal{O}_{v}^{*}, d \omega\right) \rightarrow \mathbb{C}$ as $X_{k l}^{v}(\omega)=\omega\left(E_{k l}\right)$. It is easy to see that in the non-commutative probability space $\left(M_{n}(\mathbb{C}) \otimes L^{\infty}\left(\mathcal{O}_{v}^{*}, d \omega\right)\right.$, $\operatorname{tr} \otimes d \omega)$ the random variable defined as $\sum_{k l} E_{k l} \otimes X_{k l}^{v}$ is a uniform random matrix with spectrum $\left\{v_{1}, \ldots, v_{n}\right\}$.

For a family $v(s)_{s \in S}$ (where $\left.v(s)=v_{1}(s) \eta_{1}+\cdots v_{n}(s) \eta_{n}\right)$ of elements of $\mathfrak{u}_{n}$, in the noncommutative probability space $M_{n}(\mathbb{C}) \otimes L^{\infty}\left(\prod_{s \in S} \mathcal{O}_{v(s)}^{*}, \prod_{s \in S} d \omega\right)$ the elements $\left(\sum E_{k l} \otimes X_{k l}^{v(t)}\right)_{t \in S}$ where $X_{k l}^{v(t)}\left(\prod_{s \in S} \omega_{s}\right)=\omega_{t}\left(E_{k l}\right)$ form a family of uniform random matrices with respective spectra $\left\{v_{1}(s) \ldots v_{n}(s)\right\}$, and with independent coordinates.

1.4. Following results of D. Voiculescu (see [7]), R. Speicher proved that the trace of the spectral measure of the sum of two large symmetric matrices 
is given asymptotically by the free convolution of their spectral measures, for almost all choices of the matrices with given spectrum (see [5] part 3 for the precise statement of the theorem). If one looks carefully at his arguments one can see that they imply the following result. Let $n_{m}$ be a sequence of positive integers such that $n_{m} \rightarrow \infty$ as $m \rightarrow \infty$, and for each $m$ let $X(m, s)_{s \in S}$ be a family of uniform random $n_{m} \times n_{m}$ matrices with independent entries and with respective spectra $\left\{\lambda_{1}(m, s) \ldots \lambda_{n}(m, s)\right\}$. Suppose that the measures $\frac{1}{n_{m}} \sum_{k=1}^{n_{m}} \delta_{\lambda_{k}(m, s)}$ converge weakly to some measure $\alpha(s)$ with compact support on $\mathbb{R}$. Let $D(t, m)_{t \in T}$ be families of $n_{m} \times n_{m}$ diagonal matrices which have a limit distribution as $m \rightarrow \infty$, and such that $\sup _{m}\|D(t, m)\|<\infty$ for all $t \in T$. One considers the matrices $D(t, m)$ as random matrices with constant entries. Then $\left(X(m, s)_{s \in S}\right.$, $\left.\left\{D(t, m)_{t \in T}\right\}\right)_{m \geq 0}$ is an asymptotically free family of sets of random variables.

2.1. Let $\mu \in P_{+}$and $R_{\mu}$ be as in 1.2. In this section we will prove a technical estimate which relates the moments of the random variables $\xi_{k l}^{\mu}$ and $X_{k l}^{\mu+\rho}$ defined in 1.2 and 1.3. We denote by $E$ the expectation on the (commutative) probability space $L^{\infty}\left(\mathcal{O}_{\mu+\rho}^{*}, d \omega\right)$.

Proposition 1. There exist constants $C_{r}$ depending only on $r$ such that for any sequence $\left(k_{1}, l_{1}\right), \ldots\left(k_{r}, l_{r}\right)$ in $[1, n]^{2}$ one has

$$
\left|\operatorname{tr}\left(\xi_{k_{1} l_{1}}^{\mu} \ldots \xi_{k_{r} l_{r}}^{\mu}\right)-E\left[X_{k_{1} l_{1}}^{\mu+\rho} \ldots X_{k_{r} l_{r}}^{\mu+\rho}\right]\right| \leq 6 n C_{r}(|\mu|+6 n)^{r-1} .
$$

Proof. We first give a series of lemma.

2.2. Lemma 1. For every sequence $\left(k_{1}, l_{1}\right), \ldots\left(k_{r}, l_{r}\right)$ in $[1, n]^{2}$ one has

$$
\left|\operatorname{tr}\left(\xi_{k_{1} l_{1}}^{\mu} \ldots \xi_{k_{r} l_{r}}^{\mu}\right)\right| \leq|\mu|^{r}
$$

and

$$
\left|E\left[X_{k_{1} l_{1}}^{\mu+\rho} \ldots X_{k_{r} l_{r}}^{\mu+\rho}\right]\right| \leq(|\mu|+n)^{r} .
$$

Proof. The norm of $d R_{\mu}\left(E_{k l}\right)$ on $V$ is less than $|\mu|$ so that the first inequality follows. For the second, one has $\left|X_{k l}^{\mu+\rho}\right| \leq|\mu+\rho| \leq|\mu|+n$ on $\mathcal{O}_{\mu+\rho}^{*}$ hence the second inequality.

Let us notice that the function $\prod_{k<l} \frac{\alpha_{l l}-\alpha_{k k}}{\operatorname{sh}\left(\alpha_{l l}-\alpha_{k k}\right)}$ of the variables $\alpha_{k k}$ has a convergent power series expansion in a neighbourhood of zero.

Lemma 2. Let $f\left(a_{1}, \ldots a_{n}\right)$ for $a_{1}, \ldots a_{n} \in \mathbb{N}$ be the coefficient of $\alpha_{11}^{a_{1}} \ldots \alpha_{n n}^{a_{n}}$ in the expansion of $\prod_{k<l} \frac{\alpha_{l l}-\alpha_{k k}}{\operatorname{sh}\left(\alpha_{l l}-\alpha_{k k}\right)}$. One has $\left|f\left(a_{1}, \ldots a_{n}\right)\right| \leq(6 n)^{a_{1}+\cdots+a_{n}}$. 
Proof. One has $\frac{x}{s h x}=\sum_{k=0}^{\infty}(-1)^{k} x^{2 k} \frac{E_{2 k+1}}{(2 k+1) !}$ where $E_{2 k+1}$ are Euler's numbers counting the number of alternating permutations, and $E_{2 k+1} \leq(2 k+1)$ ! hence the absolute value of the coefficient of $x^{u} y^{v}$ in $\frac{x-y}{\operatorname{sh}(x-y)}$ is $\frac{E_{u+v+1}}{(u+v+1) !} \frac{(u+v) !}{u ! v !}$ when $u+v$ is even, and 0 if $u+v$ is odd. This coefficient is thus smaller than $\frac{(u+v) !}{u ! v !}$ and hence smaller than $2^{u+v}$. We conclude from this that the coefficient of $\alpha_{11}^{a_{1}} \ldots \alpha_{n n}^{a_{n}}$ in $\prod_{k<l} \frac{\alpha_{l l}-\alpha_{k k}}{\operatorname{sh}\left(\alpha_{l l}-\alpha_{k k}\right)}$ is smaller in absolute value than that of $\alpha_{11}^{a_{1}} \ldots \alpha_{n n}^{a_{n}}$ in $\prod_{k<l} \sum_{u v} 2^{u} \alpha_{k k}^{u} 2^{v} \alpha_{l l}^{v}=\prod_{k}\left(1-2 \alpha_{k k}\right)^{-(n-1)}$. But this last coefficient is equal to $\prod_{k} 2^{a_{k}} \frac{\left(a_{k}+n\right) !}{a_{k} ! n !}$ and we can use the elementary inequalities $\frac{(u+v) !}{u ! v !} \leq(e v)^{u}$ for $v \geq 1$, and $2 e<6$, to obtain the result.

Lemma 3. For any permutation $\sigma$ of $1, \ldots, r$ one has

$$
\left|\operatorname{tr}\left(\xi_{k_{1} l_{1}}^{\mu} \ldots \xi_{k_{r} l_{r}}^{\mu}\right)-\operatorname{tr}\left(\xi_{k_{\sigma(1)} l_{\sigma(1)}}^{\mu} \ldots \xi_{k_{\sigma(r)} l_{\sigma(r)}}^{\mu}\right)\right| \leq r(r-1)|\mu|^{r-1}
$$

Proof. Let $\tau$ be a transposition of the form $(j, j+1)$ for $1 \leq j \leq r-1$. One has

$$
\begin{aligned}
& \left|\operatorname{tr}\left(\xi_{k_{1} l_{1}}^{\mu} \ldots \xi_{k_{r} l_{r}}^{\mu}\right)-\operatorname{tr}\left(\xi_{k_{\tau(1)} l_{\tau}(1)}^{\mu} \ldots \xi_{k_{\tau}(r) l_{\tau(r)}}^{\mu}\right)\right| \\
& \quad=\left|\operatorname{tr}\left(\xi_{k_{1} l_{1}}^{\mu} \ldots\left[\xi_{k_{l} l_{j}}^{\mu}, \xi_{k_{j+1} l_{j+1}}^{\mu}\right] \ldots \xi_{k_{r} l_{r}}^{\mu}\right)\right| \\
& \quad=\left|\operatorname{tr}\left(\xi_{k_{1} l_{1}}^{\mu} \ldots\left(\xi_{k_{\jmath} l_{j+1}}^{\mu} \delta_{l_{j} k_{j+1}}-\xi_{k_{j+1} l_{j}}^{\mu} \delta_{k_{\jmath} l_{j+1}}\right) \ldots \xi_{k_{r} l_{r}}^{\mu}\right)\right|
\end{aligned}
$$

since $d R_{\mu}$ is a Lie algebra representation

$$
\leq 2|\mu|^{r-1} \text { by Lemma } 1 \text {. }
$$

Since every permutation is a product of at most $\frac{r(r-1)}{2}$ such transpositions, the lemma follows.

2.3. We can now prove Proposition 1.

Thanks to the estimates of Lemmas 1 and 2 we can expand the two sides of Kirillov's formula (1) as power series of the variables $\alpha_{k l}$ in a neighbourhood of zero and equate the coefficients of both sides. Let $a=\left(a_{k l}\right)_{1 \leq k, l \leq n}$ be a sequence of nonnegative integers then the coefficient of $\prod_{k l} \alpha_{k l}^{a_{k l}}$ in the left side of (1) is equal to

$$
\sum_{\left(u_{1}, v_{1}\right), \ldots\left(u_{r}, v_{r}\right) \in \mathscr{P}_{a}} i^{r} \beta\left(\left(u_{1}, v_{1}\right), \ldots\left(u_{r}, v_{r}\right)\right) \operatorname{tr}\left(\xi_{u_{1} v_{1}}^{\mu} \ldots \xi_{u_{r} v_{r}}^{\mu}\right)
$$


where the sum is taken over the set $\mathscr{P}_{a}$ of all sequences $\left(\left(u_{1}, v_{1}\right), \ldots\left(u_{r}, v_{r}\right)\right)$ such that the number of occurences of each pair $(k, l)$ is exactly $a_{k l}$ (so that $\left.\sum_{k l} a_{k l}=r\right)$, and the numbers $\beta\left(\left(u_{1}, v_{1}\right), \ldots\left(u_{r}, v_{r}\right)\right)$ are some positive universal coefficients. Moreover, one has

$$
\sum_{\left.u=\left(u_{1}, v_{1}\right), \ldots\left(u_{r}, v_{r}\right)\right) \in \mathscr{P}_{a}} \beta(u)=\prod_{k l}\left(a_{k l} !\right)^{-1} .
$$

In the right hand side of the formula, the coefficient of $\prod_{k l} \alpha_{k l}^{a_{k l}}$ is equal to

$$
\sum_{\substack{0 \leq b_{k k} \leq a_{k k} \\ f o r} \leq k \leq n} i^{r-\sum_{k} b_{k k}} f\left(b_{11}, \ldots, b_{n n}\right) E\left[\prod_{k \neq l} \frac{\left(X_{k l}^{\mu+\rho}\right)^{a_{k l}}}{a_{k l} !} \prod_{k} \frac{\left(X_{k k}^{\mu+\rho}\right)^{a_{k k}-b_{k k}}}{\left(a_{k k}-b_{k k}\right) !}\right] .
$$

It follows that

$$
\left|\sum_{\left(u_{1}, v_{1}\right), \ldots\left(u_{r}, v_{r}\right) \in \mathscr{P}_{a}} \beta\left(\left(u_{1}, v_{1}\right), \ldots\left(u_{r}, v_{r}\right)\right) \operatorname{tr}\left(\xi_{u_{1} v_{1}}^{\mu} \ldots \xi_{u_{r} v_{r}}^{\mu}\right)-E\left[\prod_{k l} \frac{\left(X_{k l}^{\mu+\rho}\right)^{a_{k l}}}{a_{k l} !}\right]\right|
$$

is equal to

$$
\left|\sum_{\substack{0 \leq b_{k k} \leq a_{k k} \\ \text { not all } b_{k k}=0}} i^{r-\sum_{k} b_{k k}} f\left(b_{11}, \ldots, b_{n n}\right) E\left[\prod_{k \neq l} \frac{\left(X_{k l}^{\mu+\rho}\right)^{a_{k l}}}{a_{k l} !} \prod_{k} \frac{\left(X_{k k}^{\mu+\rho}\right)^{a_{k k}-b_{k k}}}{\left(a_{k k}-b_{k k}\right) !}\right]\right|
$$

which is, according to Lemmas 1 and 2, less in absolute value than

$$
\sum_{\substack{0 \leq b_{k k} \leq a_{k k} \\ \text { not all } b_{k k}=0}}(|\mu|+n)^{r-\sum_{k} b_{k k}} \prod_{k} \frac{1}{\left(a_{k k}-b_{k k}\right) !}(6 n)^{\sum_{k} b_{k k}} .
$$

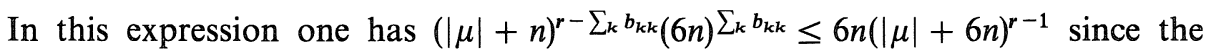
$b_{k k}$ are not all zero, so that it is less than

$$
\begin{aligned}
6 n(|\mu| & +6 n)^{r-1} \sum_{0 \leq b_{k k} \leq a_{k k}} \prod_{k} \frac{1}{\left(a_{k k}-b_{k k}\right) !} \\
& =6 n(|\mu|+6 n)^{r-1} \prod_{k}\left(\sum_{w=0}^{a_{k k}} \frac{1}{w !}\right) \\
& \leq 6 n(|\mu|+6 n)^{r-1} \prod_{\substack{k \\
a_{k k} \neq 0}} e \\
& \leq 6 n(|\mu|+6 n)^{r-1} e^{r}
\end{aligned}
$$

since there are at most $r$ numbers $a_{k k} \neq 0$.

For any sequence $\left(k_{1}, l_{1}\right), \ldots,\left(k_{r}, l_{r}\right)$ which belongs to $\mathscr{P}_{a}$ one has $E\left[X_{k_{1} l_{1}}^{\mu+\rho} \ldots X_{k_{r} l_{r}}^{\mu+\rho}\right]=E\left[\prod_{k l}\left(X_{k l}^{\mu+\rho}\right)^{a_{k l}}\right]$ and, by Lemma 3, 


$$
\begin{aligned}
& \left|\prod_{k l}\left(a_{k l} !\right)^{-1} \operatorname{tr}\left(\xi_{k_{1} l_{1}}^{\mu} \ldots \xi_{k_{r} l_{r}}^{\mu}\right)-\sum_{u=\left(\left(u_{1}, v_{1}\right), \ldots\left(u_{r}, v_{r}\right)\right) \in \mathscr{P}_{a}} \beta(u) \operatorname{tr}\left(\xi_{u_{1} v_{1}}^{\mu} \ldots \xi_{u_{r} v_{r}}^{\mu}\right)\right| \\
& \quad \leq \sum_{u=\left(\left(u_{1}, v_{1}\right), \ldots\left(u_{r}, v_{r}\right)\right) \in \mathscr{P}_{a}} \beta(u)\left|\operatorname{tr}\left(\xi_{k_{1} l_{1}}^{\mu} \ldots \xi_{k_{r} l_{r}}^{\mu}\right)-\operatorname{tr}\left(\xi_{u_{1} v_{1}}^{\mu} \ldots \xi_{u_{r} v_{r}}^{\mu}\right)\right| \\
& \quad \leq \prod_{k l}\left(a_{k l} !\right)^{-1} r(r-1)|\mu|^{r-1} .
\end{aligned}
$$

From the majorizations of the quantities (2) and (3) it follows that

$$
\begin{aligned}
& \left|\operatorname{tr}\left(\xi_{k_{1} l_{1}}^{\mu} \ldots \xi_{k_{r} l_{r}}^{\mu}\right)-E\left[X_{k_{1} l_{1}}^{\mu+\rho} \ldots X_{k_{r} l_{r}}^{\mu+\rho}\right]\right| \\
& \quad \leq r(r-1)|\mu|^{r-1}+\prod_{k l}\left(a_{k l} !\right) 6 n e^{r}(|\mu|+6 n)^{r-1} \\
& \quad \leq r(r-1)|\mu|^{r-1}+6 n r ! e^{r}(|\mu|+6 n)^{r-1}
\end{aligned}
$$

and Proposition 1 follows easily from this estimate.

2.4. We have the following corollary of Proposition 1.

Corollary. Let $\mu^{m}$ be a sequence in $P_{+}$and $\varepsilon_{m}$ a sequence in $\mathbb{R}_{+}$such that $\varepsilon_{m} \rightarrow 0$ and $\varepsilon_{m} \mu^{m} \rightarrow v \in \mathfrak{D}_{n}^{*}$ as $m \rightarrow \infty$. Then the family of random variables $\left(\varepsilon_{m} \xi_{k l}^{\mu^{m}}\right)_{1 \leq k, l \leq n}$ converge in distribution to $\left(X_{k l}^{v}\right)_{1 \leq k, l \leq n}$ as $m \rightarrow \infty$.

Proof. It is enough to prove that for any sequence $\left(k_{1}, l_{1}\right), \ldots\left(k_{r}, l_{r}\right)$ in $[1, n]^{2}$ one has

$$
\operatorname{tr}\left(\varepsilon_{m} \xi_{k_{1} l_{1}}^{\mu^{m}} \ldots \varepsilon_{m} \xi_{k_{r} l_{r}}^{\mu^{m}}\right) \rightarrow E\left[X_{k_{1} l_{1}}^{v} \ldots X_{k_{r} l_{r}}^{v}\right]
$$

as $m \rightarrow \infty$. From Proposition 1 we have

$$
\left|\operatorname{tr}\left(\varepsilon_{m} \xi_{k_{1} l_{1}}^{\mu^{m}} \ldots \varepsilon_{m} \xi_{k_{r} l_{r}}^{\mu^{m}}\right)-E\left[\varepsilon_{m} X_{k_{1} l_{1}}^{\mu^{m}+\rho} \ldots \varepsilon_{m} X_{k_{r} l_{r}}^{\mu^{m}+\rho}\right]\right| \leq\left(\varepsilon_{m}\right)^{r} 6 n C_{r}\left(\left|\mu^{m}\right|+6 n\right)^{r-1}
$$

and the expression on the right goes to 0 as $m \rightarrow \infty$. Moreover

$$
\begin{aligned}
& E\left[\varepsilon_{m} X_{k_{1} l_{1}}^{\mu^{m}+\rho} \ldots \varepsilon_{m} X_{k_{r} l_{r}}^{\mu^{m}+\rho}\right]=\int_{\mathcal{O}_{\mu}^{*}{ }_{+}^{*}+\rho} \varepsilon_{m} X_{k_{1} l_{1}}^{\mu^{m}+\rho}(\omega) \ldots \varepsilon_{m} X_{k_{r} l_{r}}^{\mu^{m}+\rho}(\omega) d \omega
\end{aligned}
$$

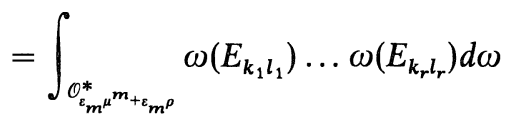

and since $\varepsilon_{m} \mu^{m} \rightarrow v$ this last expression converges as $m \rightarrow \infty$ to

$$
\int_{\mathcal{O}_{v}^{*}} \omega\left(E_{k_{1} l_{1}}\right) \ldots \omega\left(E_{k_{r} l_{r}}\right) d \omega=E\left[X_{k_{1} l_{1}}^{v} \ldots X_{k_{r} l_{r}}^{v}\right] .
$$

This proves the corollary. 


\section{$\S 3$}

In this section we will let the dimension $n$ go to infinity.

3.1. Let $R$ be a finite dimensional unitary representation of $U(n)$. We will associate to $R$ two objects, namely a probability measure on $\mathbb{Z}$ and a non commutative random variable.

We define the measure $\mathscr{M}(R)$ in the following way, if $R$ is irreducible with highest weight $\mu=\sum \mu_{k} \eta_{k}$ then $\mathscr{M}(R)=\frac{1}{n} \sum_{k} \delta_{\mu_{k}}$. If $R$ can be decomposed into a sum of two subrepresentations $R=R_{1} \oplus R_{2}$ then $\mathscr{M}(R)$ is the convex combination $\mathscr{M}(R)=\frac{\operatorname{dim}\left(R_{1}\right)}{\operatorname{dim}(R)} \mathscr{M}\left(R_{1}\right)+\frac{\operatorname{dim}\left(R_{2}\right)}{\operatorname{dim}(R)} \mathscr{M}\left(R_{2}\right)$. Thanks to the complete reducibility of representations of $U(n)$, there is a uniquely defined map $R \mapsto \mathscr{M}(R)$ from finite dimensional representations of $U(n)$ to probability measures on $\mathbb{Z}$ satisfying these requirements.

Let $V$ be the space of the representation of $R$, which is a Hilbert space. The $C$-random variable associated to $R$ is the element of the non-commutative probability space $\left(M_{n}(\mathbb{C}) \otimes \mathscr{L}(V), t r\right)$ (where $t r$ is the normalized trace) defined as $C(R)=\sum_{k l} E_{k l} \otimes d R\left(E_{k l}\right)$ where $d R$ is the representation of the Lie algebra $g l_{n}(\mathbb{C})$ corresponding to $R$. We use the letter $C$ because of the obvious relation with Casimir operator.

It is easy to see that $C(R)$ is a self-adjoint element of $M_{n}(\mathbb{C}) \otimes \mathscr{L}(V)$, hence it has a distribution which is given by a probability measure on $\mathbb{R}$, which is a finite convex combination of Dirac measures. It turns out that this measure, although not equal to $\mathscr{M}(R)$ is closely related to it. In fact it follows easily from the corollary of Proposition 1, proved in Section 2 that if $\mu^{m}$ is a sequence in $P_{+}$and $\varepsilon_{m}$ a sequence in $\mathbb{R}_{+}$such that $\varepsilon_{m} \rightarrow 0$ and $\varepsilon_{m} \mu^{m} \rightarrow v \in \mathrm{D}_{n}^{*}$ as $m \rightarrow \infty$ if $R_{m}$ is an irreducible representation with highest weight $\mu^{m}$ then the distribution of $\varepsilon_{m} C\left(R_{m}\right)$ and the measure $\mathscr{M}^{\varepsilon_{m}}\left(R_{m}\right)$ converge to the same limit which is $\frac{1}{n} \sum_{k} \delta_{v_{k}}$.

3.2. The explicit distribution of $C(R)$ can be obtained. Indeed, the computations of Zelobenko (see [8] Ch IX, 60) show that the operator $C(R)$ has the eigenvalues $\lambda_{j}=\mu_{j}+n-j$ for $j=1, \ldots, n$ the multiplicity of $\lambda_{j}$ being $\prod_{k \neq j} \frac{\mu_{j}-j-\mu_{k}+k-1}{j-k}$.

Another method of obtaining this result was communicated to us by Patrick Polo [3] and is as follows. On the space $\mathbb{C}^{n} \otimes V$ let $U(n)$ act by the representation $\chi: g \mapsto \bar{g} \otimes R(g)$. A simple computation reveals that the matrix $C(R)$ commutes with all the operators of this representation. The representation $\chi$ is the tensor product of the representation $R$ and of the conjugate of 
the basic representation of $U(n)$. It is well known (since the highest weight of the conjugate representation is a minuscule weight) that this representation decomposes into a sum of the irreducible representations with highest weights $\mu-\eta_{k}$ for the numbers $k \in\{1, \ldots, n\}$ such that $\mu-\eta_{k}$ is a dominant weight. From this we conclude that each of the subspaces of these representations is an eigenspace for $C(R)$. The corresponding eigenvalue can be computed by evaluating the image by $C(R)$ of a highest weight vector.

We could have defined the measure $\mathscr{M}(R)$ as being the distribution of $C(R)$, and the theorem that we prove in Section 3.4, as well as Proposition 3 would be true and have simpler proofs. However, the formula giving the law of $C(R)$ is complicated so that we have preferred the simpler definition of $\mathscr{M}(R)$ given in the text.

We will not use the result of this section in the sequel.

3.3. We shall now prove the following proposition which deals with the case when the dimension goes to infinity and the representations are allowed to be reducible.

Proposition 2. Let $n_{m}$ be a sequence of positive integers such that $n_{m} \rightarrow \infty$. For each $m \geq 1$, let $R_{m}$ be a representation of $U\left(n_{m}\right)$. Let $\varepsilon_{m}$ be a sequence in $\mathbb{R}_{+}$such that

i) $\varepsilon_{m}\left(n_{m}\right)^{r} \rightarrow 0$ for all $r \in \mathbb{N}$

ii) there is a constant $c$ such that for every $m$ and every highest weight $\mu$ occuring in the representation $R_{m}$ one has $\varepsilon_{m}|\mu| \leq c$

iii) the measures $\mathscr{M}^{\varepsilon_{m}}\left(R_{m}\right)$ have a limit $\alpha$ as $m \rightarrow \infty$, then the distribution of $\varepsilon_{m} C\left(R_{m}\right)$ converges to $\alpha$ as $m \rightarrow \infty$.

Proof. First remark that by condition ii) the distribution $\alpha$ must come from a probability measure with compact support on $[-c, c]$.

We must show that for all $r \in \mathbb{N}, \operatorname{tr}\left(\left(\varepsilon_{m} C\left(R_{m}\right)\right)^{r}\right) \rightarrow \int_{\mathbb{R}} x^{r} \alpha(d x)$.

For each $m$ let $R_{m}=R_{m}^{(1)} \oplus R_{m}^{(2)} \oplus \cdots \oplus R_{m}^{\left(u_{m}\right)}$ be a decomposition of $R_{m}$ into irreducible components with highest weights $\mu_{m}^{w}$ for $w=1, \ldots, u_{m}$, then $C\left(R_{m}\right)=\pi_{1} C_{1} \pi_{1}+\cdots+\pi_{u_{m}} C_{u_{m}} \pi_{u_{m}}$ where the $\pi_{w}$ are orthogonal self-adjoint projections of trace $\operatorname{tr}\left(\pi_{w}\right)=\frac{\operatorname{dim}\left(R_{m}^{(w)}\right)}{\operatorname{dim}\left(R_{m}\right)}$, and each $C_{w}$ is an operator with the same distribution as $C\left(R_{m}^{(w)}\right)$, and so $\operatorname{tr}\left(C\left(R_{m}\right)^{r}\right)=\sum_{w=1}^{u_{m}} \frac{\operatorname{dim}\left(R_{m}^{(w)}\right)}{\operatorname{dim}\left(R_{m}\right)} \operatorname{tr}\left(C\left(R_{m}^{(w)}\right)^{r}\right)$. Since $\mathscr{M}\left(R_{m}\right)=\sum_{w=1}^{u_{m}} \frac{\operatorname{dim} R_{m}^{(w)}}{\operatorname{dim}\left(R_{m}\right)} \mathscr{M}\left(R_{m}^{(w)}\right)$ it is enough to show that for each $w \in$ $\left[1, u_{m}\right]$ one has $\varepsilon_{m}^{r}\left|\operatorname{tr}\left(C\left(R_{m}^{(w)}\right)^{r}\right)-\int_{\mathbb{R}} x^{r} \mathscr{M}\left(R_{m}^{(w)}\right)(d x)\right| \leq v_{m}$ where $v_{m}$ does not depend on $w$ and converges to zero as $m \rightarrow \infty$. 
One has

$$
\operatorname{tr}\left(C\left(R_{m}^{(w)}\right)^{r}\right)=\frac{1}{n_{m}} \sum_{1 \leq k_{1}, \ldots, k_{r} \leq n_{m}} \operatorname{tr}_{w}\left(\xi_{k_{1} k_{2}}^{\mu_{m}^{w}} \xi_{k_{2} k_{3}}^{\mu_{m}^{w}} \ldots \xi_{k_{r} k_{1}}^{\mu_{m}^{w}}\right)
$$

where $t r_{w}$ is the normalized trace on the representation space of $R_{m}^{(w)}$.

Let $M_{\mu_{m}^{w}+\rho}=\sum_{k l} E_{k l} X_{k l}^{\mu_{m}^{w}+\rho}$ where $X_{k l}^{\mu_{m}^{w}+\rho}$ are as in 1.2.

When computing $E\left[\operatorname{tr}\left(\left(M_{\mu_{m}^{w}+\rho}\right)^{r}\right]\right.$ one can expand the trace with respect to the variables $X_{k l}^{\mu_{m}^{w}+\rho}$ to get the expression

$$
\frac{1}{n_{m}} \sum_{1 \leq k_{1}, \ldots, k_{r} \leq n_{m}} E\left(X_{k_{1} k_{2}}^{\mu_{m}^{w}+\rho} X_{k_{2} k_{3}}^{\mu_{m}^{w}+\rho} \ldots X_{k_{r} k_{1}}^{\mu_{m}^{w}+\rho}\right)
$$

It follows then from Proposition 1 that

$$
\left|\operatorname{tr}\left(C\left(R_{m}^{(w)}\right)^{r}\right)-E\left[\operatorname{tr}\left(\left(M_{\mu_{m}^{w}+\rho}\right)^{r}\right]\right)\right| \leq\left(n_{m}\right)^{r-1} 6 n_{m} C_{r}\left(\left|\mu_{m}^{w}\right|+6 n_{m}\right)^{r-1} .
$$

Since $M_{\mu_{m}^{w}+\rho}$ is a uniform random matrix with spectrum $\left(\mu_{m}^{w}\left(i E_{j j}\right)+n_{m}-\right.$ $\left.j+1, j=1, \ldots, n_{m}\right)$, and since $\mathscr{M}\left(R_{m}^{(w)}\right)=\sum_{j} \delta_{\mu_{m}^{w}\left(i E_{j j}\right)}$, using the elementary inequality $\left|(a+b)^{r}-a^{r}\right| \leq r|b|(|a|+|b|)^{r-1}$ for $a, b \in \mathbb{R}$ one has

$$
\operatorname{tr}\left(\left(M_{\mu_{m}^{w}+\rho}\right)^{r}\right)=\frac{1}{n_{m}} \sum_{j}\left(\mu_{m}^{w}\left(i E_{j j}\right)+n_{m}-j+1\right)^{r}=\int_{\mathbb{R}} x^{r} d \mathscr{M}\left(R_{m}^{w}\right)(x)+g(m, w)
$$

with $|g(m, w)| \leq r n_{m}\left(\left|\mu_{m}^{w}\right|+n_{m}\right)^{r-1}$. We see that for every $w$

$$
\begin{aligned}
& \left|\left(\varepsilon_{m}\right)^{r}\left(\operatorname{tr}\left(C\left(R_{m}^{(w)}\right)^{r}\right)-\int x^{r} d \mathscr{M}\left(R_{m}^{(w)}\right)(x)\right)\right| \\
& \quad \leq\left(\varepsilon_{m}\right)^{r}\left(n_{m}\right)^{r-1}\left(6 n_{m} C_{r}\left(\left|\mu_{m}^{w}\right|+6 n_{m}\right)^{r-1}+r n_{m}\left(\left|\mu_{m}^{w}\right|+n_{m}\right)^{r-1}\right) \\
& \quad \leq \varepsilon_{m}\left(n_{m}\right)^{r-1}\left(6 n_{m} C_{r}\left(c+6 \varepsilon_{m} n_{m}\right)^{r-1}+r n_{m}\left(c+\varepsilon_{m} n_{m}\right)^{r-1}\right) .
\end{aligned}
$$

This quantity is independent of $w$ and goes to 0 by hypothesis $i$ ). This finishes the proof.

3.4. We say that a family of representations $R(s)$ (indexed by some finite set $S$ ) of $U(n)$ on a finite dimensional space $V$ is independent if there exists a tensor product decomposition $V=V_{0} \otimes\left(\otimes_{s \in S} V_{s}\right)$ such that for every $t \in S$ and $g \in U(n)$ one has $R(t)(g)=I d_{V_{0}} \otimes\left(\otimes_{s \neq t} I d_{V_{s}}\right) \otimes \widetilde{R}(t)(g)$ where $\widetilde{R}(t)$ is a representation on $V_{t}$.

For independent representations one has $\left[d R(s)\left(E_{k l}\right), d R\left(s^{\prime}\right)\left(E_{k^{\prime} l^{\prime}}\right)\right]=0$ for all $k, l, k^{\prime}, l^{\prime}$ if $s \neq s^{\prime}$, and the trace factorizes:

$$
\operatorname{tr}_{V}\left(\prod_{s \in S} \zeta_{s}\right)=\prod_{s \in S} t_{V}\left(\zeta_{s}\right)
$$

where $\zeta_{s}$ belongs to the algebra generated by the operators $d R(s)\left(E_{k l}\right)$.

We are now in position to state and prove the main result of this paper. 
Theorem. Let $S$ be a finite set. Let $n_{m}$ be a sequence of positive integers such that $n_{m} \rightarrow \infty$. Let, for each $m \geq 0, R_{m}(s), s \in S$ be a family of independent isotypic representations of $U\left(n_{m}\right)$ (i.e. the representations $\widetilde{R}_{m}(s)$ are irreducible), and let $\varepsilon_{m}, m \geq 0$ be a sequence in $\mathbb{R}_{+}$such that

i) for every $r \in \mathbb{N} \varepsilon_{m}\left(n_{m}\right)^{r} \rightarrow 0$

ii) there is a constant $c$ such that $\varepsilon_{m}\left|\mu^{m}(s)\right| \leq c$ for all $m \in \mathbb{N}, s \in S$ where $\mu^{m}(s)$ is the highest weight of $R_{m}(s)$

iii) there exists probability distributions $\alpha(s)$ such that $\mathscr{M}^{\varepsilon_{m}}\left(R_{m}(s)\right) \rightarrow \alpha(s)$.

Let furthermore $D(t, m)_{t \in T}$ be families of $n_{m} \times n_{m}$ diagonal matrices which have a limit distribution as $m \rightarrow \infty$ and such that $\sup _{m}\|D(t, m)\|<\infty$ for all $t \in T$. We consider them as elements of $M_{n_{m}}(\mathbb{C}) \otimes \mathscr{L}(V)$ by tensoring with $I d_{V}$.

Then $\left(C\left(R_{m}(s)\right)_{s \in S},\left\{D(t, m)_{t \in T}\right\}\right)$ is an asymptotically free family of sets of random variables.

Proof. As in [7] we can assume that the matrices $D(t, m)_{t \in T}$ form a multiplicative semi-group and that the identity is among them. We will study the asymptotic evaluation of quantities like

$$
\operatorname{tr}\left(D ( t _ { 1 } , m ) \left(\varepsilon_{m} C\left(R_{m}\left(s_{1}\right)\right)^{r_{1}} \ldots D\left(t_{l}, m\right)\left(\varepsilon_{m} C\left(R\left(s_{l}\right)\right)^{r_{l}} D\left(t_{l+1}, m\right)\right)\right.\right.
$$

for $t_{1}, \ldots, t_{l+1} \in T, s_{1}, \ldots, s_{l} \in S$ and $r_{1} \ldots r_{l} \in \mathbb{N}$.

Expanding this trace as in the proof of Proposition 2, and using the fact that the representations $R_{m}(s)$ are independent we see that it is equal to

$$
\sum_{u \in \mathscr{S}} d(u) H(u)
$$

where $\mathscr{S}$ is a certain set of maps of $S \cup T$ into sequences of the form

$$
\left(\left(u_{1}(s), v_{1}(s)\right) \ldots,\left(u_{z(s)}(s), v_{z(s)}(s)\right)\right)
$$

so that $z(s)=\sum_{s_{k}=s} r_{k}$ for $s \in \mathscr{S}$ and $z(t)=\sum_{t_{k}=t} 1$ for $t \in T$, one has card $(\mathscr{S}) \leq$ $n_{m}^{r_{1}+\cdots+r_{l}+l+1}$ and for each $u \in \mathscr{S}$,

$$
d(u)=\prod_{t \in T} \prod_{k=1}^{z(t)} D(t, m)_{u_{k}(t) v_{k}(t)}
$$

and

$$
H(u)=\prod_{s \in S} \operatorname{tr}_{V}\left(\varepsilon_{m} \xi_{u_{1}(s) v_{1}(s)}^{\mu_{m}(s)} \ldots \varepsilon_{m} \xi_{u_{z(s)}(s) v_{z(s)}(s)}^{\mu_{m}(s)}\right)
$$

Let $M_{\mu^{m}(s)+\rho}$ be independent random matrices as in the proof of Proposition 2. Expanding the trace in the expression

$$
\operatorname{tr}\left(D\left(t_{1}, m\right)\left(\varepsilon_{m} M_{\mu^{m}\left(s_{1}\right)+\rho}\right)^{r_{1}} \ldots D\left(t_{l}, m\right)\left(\varepsilon_{m} M_{\mu^{m}\left(s_{l}\right)+\rho}\right)^{r_{l}} D\left(t_{l+1}, m\right)\right)
$$

we see that it is equal to

$$
\sum_{u \in \mathscr{S}} d(u) G(u)
$$


where $G(u)$ is the expression obtained from $H(u)$ by replacing $\xi_{k l}^{\mu_{m}(s)}$ by $M_{k l}^{\mu^{m}(s)+\rho}$. Using the estimates of Proposition 1 and Lemma 1 in the same way as in the proof of Proposition 2, and the fact that $\varepsilon_{m}\left|\mu_{m}(s)\right| \leq c$ and $\sup _{m}\|D(t, m)\| \leq$ $c(t)$ for some constants $c(t)$, we can see that $|H(u)-G(u)|$ is bounded by $\varepsilon_{m} K_{m}$ where $K_{m}$ is a polynomial in $n_{m}$ whose degree and coefficients depend only on $c, l, r_{1} \ldots r_{l}$ and the constants $c(t)$. Details are straightforward and left to the reader. We deduce from this that

$$
\sum_{u \in \mathscr{S}} d(u)|G(u)-H(u)| \leq \varepsilon_{m} n_{m}^{r_{1}+\cdots+r_{l}+l+1} K_{m}
$$

and by hypothesis $i$ ) this quantity goes to zero as $m \rightarrow \infty$.

Thanks to the result of Section 1.4, the expression

$$
\operatorname{tr}\left(D\left(t_{1}, m\right)\left(\varepsilon_{m} M_{\mu^{m}\left(s_{1}\right)+\rho}\right)^{r_{1}} \ldots D\left(t_{l}, m\right)\left(\varepsilon_{m} M_{\mu^{m}\left(s_{l}\right)+\rho}\right)^{r_{l}} D\left(t_{l+1}, m\right)\right)
$$

has a limit which is

$$
\phi\left(D\left(t_{1}\right) X\left(s_{1}\right)^{r_{1}} D\left(t_{2}\right) \ldots X\left(s_{l}\right)^{r_{l}} D\left(t_{l+1}\right)\right)
$$

where $(X(s) s \in S,\{D(t), t \in T\})$ is a free family in some non commutative probability space $(\mathscr{A}, \phi)$ and the law of $X(s)$ is $\alpha(s)$, the law of $\{D(t), t \in T\}$ is the limit of the law of $\{D(t, m), t \in T\}$. So we have proved that

$$
\operatorname{tr}\left(D ( t _ { 1 } , m ) \left(\varepsilon_{m} C\left(R_{m}\left(s_{1}\right)\right)^{r_{1}} \ldots D\left(t_{l}, m\right)\left(\varepsilon_{m} C\left(R\left(s_{l}\right)\right)^{r_{l}} D\left(t_{l+1}, m\right)\right)\right.\right.
$$

also converges towards

$$
\phi\left(D\left(t_{1}\right) X\left(s_{1}\right)^{r_{1}} D\left(t_{2}\right) \ldots X\left(s_{l}\right)^{r_{l}} D\left(t_{l+1}\right)\right)
$$

and this finishes the proof of the theorem.

We will now apply the preceding theorem to the problem of decompositions of tensor products and of restrictions.

Proposition 3. Let $n_{m}$ be a sequence of positive integers such that $n_{m} \rightarrow \infty$. For each $m \geq 1$ let $R_{m}(1)$ and $R_{m}(2)$ be two irreducible representations with highest weights $\mu^{m}(1)$ and $\mu^{m}(2)$, such that $\mathscr{M}^{\varepsilon_{m}}\left(R_{m}(s)\right) \rightarrow \alpha(s)$ as $m \rightarrow \infty$ for $s=1,2$, where $\alpha(1)$ and $\alpha(2)$ are two probability measures with compact support. Suppose that the sequences $\varepsilon_{m}\left|\mu^{m}(s)\right|$ are bounded.

Then $\mathscr{M}^{\varepsilon_{m}}\left(R_{m}(1) \otimes R_{m}(2)\right) \rightarrow \alpha(1) \oplus \alpha(2)$.

Put $R_{m}=R_{m}(1)$ and $\alpha=\alpha(1)$. Let $q_{m}$ be a sequence of integers such that $q_{m} \leq n_{m}$ and $\left.\left.\frac{q_{m}}{n_{m}} \rightarrow p \in\right] 0,1\right]$, and call $T_{m}$ the restriction of $R_{m}$ to the subgroup $U\left(q_{m}\right)$ of $U\left(n_{m}\right)$ (imbedded as acting on the $q_{m}$ first vectors of the canonical basis of $\left.\mathbb{C}^{n_{m}}\right)$.

Then $\mathscr{M}^{\varepsilon_{m}}\left(T_{m}\right)$ converges towards the measure $\frac{1}{p}\left(\alpha \otimes\left(p \delta_{1}+(1-p) \delta_{0}\right)-\right.$ $\left.(1-p) \delta_{0}\right)$, where $\otimes$ denotes the free multiplicative convolution. 
Proof. The representations $R_{m}(1) \otimes I d$ and $I d \otimes R_{m}(2)$ acting on the space of the representation $R_{m}(1) \otimes R_{m}(2)$ are independent representations, so that the random variable $C\left(R_{m}(1) \otimes R_{m}(2)\right)$ can be written as $C_{m}(1)+C_{m}(2)$ where $\varepsilon_{m} C_{m}(1)$ and $\varepsilon_{m} C_{m}(2)$ have the same distribution respectively as $\varepsilon_{m} C\left(R_{m}(1)\right)$ and $\varepsilon_{m} C\left(R_{m}(2)\right)$ and are asymptotically free by the preceding theorem. We deduce from this that the law of $\varepsilon_{m} C\left(R_{m}(1) \otimes R_{m}(2)\right)$ converges towards the free convolution of $\alpha(1)$ and $\alpha(2)$. Applying Proposition 2 to the sequence of random variables $\varepsilon_{m} C\left(R_{m}(1) \otimes R_{m}(2)\right)$ we see that the sequence $\mathscr{M}^{\varepsilon_{m}}\left(R_{m}(1) \otimes R_{m}(2)\right)$ converges towards $\alpha(1) \oplus \alpha(2)$ (we can apply Proposition 2 because all the highest weights occuring in $R_{m}(1) \otimes R_{m}(2)$ have components smaller than $\left|\mu^{m}(1)\right|+$ $\left.\left|\mu^{m}(2)\right|\right)$.

Let $D(m)$ be the diagonal matrix $\sum_{k=1}^{q_{m}} E_{k k}$ then $D(m)$ has a limit distribution which is $(1-p) \delta_{0}+p \delta_{1}$ and by the theorem it is asymptotically free with the random variable $\varepsilon_{m} C\left(R_{m}\right)$. Now we see that $D(m) C\left(R_{m}\right) D(m)$ is the upper left corner embedding of the matrix $C\left(T_{m}\right)$ (which lies in $M_{q_{m}}(\mathbb{C}) \otimes \mathscr{L}(V)$ ) in the space $M_{n_{m}}(\mathbb{C}) \otimes \mathscr{L}(V)$. We deduce from this that the distribution of $C\left(T_{m}\right)$ can be obtained from that of $D(m) C\left(R_{m}\right) D(m)$ by substracting $\left(1-\frac{q_{m}}{n_{m}}\right) \delta_{0}$ and then multiplying by $\frac{n_{m}}{q_{m}}$. But the limit distribution of $D(m) C\left(R_{m}\right) D(m)$ is the free multiplicative convolution of $(1-p) \delta_{0}+p \delta_{1}$ and $\alpha$, so the result follows again by an application of Proposition 2 .

3.5. We will now give an explicit example. Let us consider the group $U(n)$ and the dominant weight $(K, \ldots, K, 0, \ldots, 0)$ where the first $l$ coordinates are $K$ 's and the remaining are 0's. Proceeding recursively, and using Weyl's branching rule, we see that for $m$ smaller than $l$ and $n-l$, the restriction of the corresponding representation $R_{m}$ to $U(n-m)$ is the sum of the representations with highest weights $\left(K, \ldots, K, \varphi_{1}, \ldots \varphi_{m}, 0 \ldots, 0\right)$ where $K \geq \varphi_{1} \geq \cdots \geq$ $\varphi_{m} \geq 0$ and there are $K$ 's on the first $l-m$ coordinates and 0 's on the last $n-l-m$, these representations occurring with multiplicity $\prod_{k<l} \frac{\varphi_{k}-\varphi_{l}+l-k}{l-k}$. This fact can also be established by decomposing the restriction of the representation to the subgroup $U(m) \times U(n-m) \subset U(n)$.

If $n \rightarrow \infty$ and $\left.\left.\frac{l}{n} \rightarrow p \in[0,1], \frac{m}{n} \rightarrow q \in\right] 0,1\right]$, and $K n^{-r} \rightarrow 0$ then the measure $\mathscr{M}^{1 / K}\left(R_{n}\right)$ converges to $(1-p) \delta_{0}+p \delta_{1}$ and by Proposition 3 , the measure $\mathscr{M}^{1 / K}\left(T_{n}\right)$, where $T_{n}$ is the restriction of $R_{n}$ to $U(n-m)$ converges towards

$$
\frac{1}{q}\left[\left((1-p) \delta_{0}+p \delta_{1}\right) \otimes\left(q \delta_{0}+(1-q) \delta_{1}\right)-(1-q) \delta_{0}\right]
$$


In particular let us take $n=2 l=2 m$ then the limit measure $\left(\frac{1}{2} \delta_{0}+\frac{1}{2} \delta_{1}\right) \otimes$ $\left(\frac{1}{2} \delta_{0}+\frac{1}{2} \delta_{1}\right)-\frac{1}{2} \delta_{0}$ is the arcsine law given by the distribution function $F(t)=$ $\frac{2}{\pi}$ Arcsine $\sqrt{t}$ on $[0,1]$ (see [1] Example 3.6.7). Since we have convergence of all moments and the limit distribution function is continuous, we have convergence of the distribution function of the measure $\mathscr{M}^{1 / K}\left(T_{n}\right)$. The distribution function of the measure $\mathscr{M}^{1 / K}\left(T_{n}\right)$ can be computed. Indeed, we know that $T_{n}$ decomposes into the sum of the irreducible representations of highest weights $\left(\varphi_{1}, \ldots, \varphi_{m}\right)$ with multiplicity $\prod_{1 \leq k<l \leq m} \frac{\varphi_{k}-\varphi_{l}+l-k}{l-k}$. By Weyl's formula, the dimension of this representation is also $\prod_{1 \leq k<l \leq m} \frac{\varphi_{k}-\varphi_{l}+l-k}{l-k}$ and the dimension of $R_{n}$ is $\prod_{1 \leq k, l \leq m} \frac{K+m+l-k}{m+l-k}$, hence

$$
\begin{aligned}
& \mathscr{M}^{1 / K}\left(T_{n}\right) \\
& \quad=\left(\prod_{1 \leq k, l \leq m} \frac{m+l-k}{K+m+l-k}\right) \sum_{K \geq \varphi_{1} \geq \cdots \geq \varphi_{m} \geq 0}\left(\prod_{k<l} \frac{\varphi_{k}-\varphi_{l}+l-k}{l-k}\right)^{2} \frac{1}{m} \sum_{j=1}^{m} \delta_{\varphi_{k} / K} .
\end{aligned}
$$

Since $K n^{-3} \rightarrow 0$ one has $\prod_{1 \leq k, l \leq m} \frac{m+l-k}{K+m+l-k} \sim K^{-m^{2}}\left(\prod_{1 \leq k, l \leq n} m+l-k\right)$ as $n=2 m \rightarrow \infty$ and the convergence of the distribution function shows that for every $t \in[0,1]$

$$
\begin{aligned}
K_{K \geq \varphi_{1} \geq \cdots \geq \varphi_{m} \geq 0} & \left(\prod_{k<l} \frac{\varphi_{k}-\varphi_{l}+l-k}{l-k}\right)^{2} \frac{1}{m} \operatorname{card}\left\{j \mid \varphi_{j} \leq t K\right\} \\
& =\left(\frac{K^{m^{2}}}{\prod_{1 \leq k, l \leq m} m+l-k}\right)\left(\frac{2}{\pi} \operatorname{Arcsine} \sqrt{t}+o(1)\right)
\end{aligned}
$$

as $m \rightarrow \infty$.

\section{References}

[1] Dykema, K. J., Nica, A. and Voiculescu, D. V., Free random variables, CRM Monograph series, 1, Centre de Recherches Mathématiques, Université de Montréal, 1991.

[2] Kirillov, A. A., The characters of unitary representations of Lie groups, Funct. Anal. Appl., 2 (1968), 133-146.

[3] Polo, P., private communication.

[4] Robinson, G. de B., Representation theory of the symmetric group, University of Toronto Press, 1961. 
[5] Speicher, R., Free convolution and the random sum of matrices, Publ. RIMS Kyoto Univ., 29 (1993), 731-744.

[6] Steinberg, R., A generalized Clebsch-Gordan theorem, Bull. Amer. Math. Soc., 67 (1961), 406-407.

[7] Voiculescu, D., Limit laws for random matrices and free products, Invent. Math., 104 (1991), 201-220.

[8] Zelobenko, D. P., Compact Lie groups and their representations, Trans. Math. Monographs, 40, AMS, Providence, Rhode Island, 1973. 
